LA-UR $-90-3328$

TITLE: State Space Forecasting and Noise Reduction

AUTHOR(S): Stephen Eubank, Martin Casdagli, J. Doyne Farmer, John Gibson

Los Alamos National Laboratory

$L A-U R--90-3328$

DE91 000184

SUBMITTED TO: IEEE Conference on Decision \& Control Honolulu, Hawai, 1990

\title{
DISCLAIMER
}

This report was prepared as an account of work sponsored by an agency of the United States Government. Neither the United States Government nor any agency thereof, nor any of their employees, makes any warranty, express or implied, or assumes at y legal liability or responsibility for the accuracy, completeness, or usefulness of any information, apparatus, product, or process disclused, or represents that its use would not infringe privately owned rigi!'s Reference herein to any specific commercial product, process, or service by trade name, trademark, manufacturer, or otherwise does not necessarily constitute or imply its endorsement, recommendation, or favoring by the United States (jovernment or any agency thereof. The vie"*s and opinions of authors expressed herein do not necessarily state or reflect those of the United States Covernment or any agency thereof.

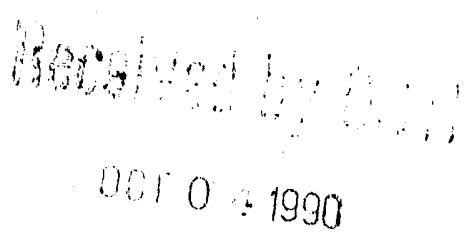

By acceplance of this article the publisher recognizes that the US Government retains a nonexclusive. royaliy-free license io publish or reprodure the published form of this contribution. or 10 allow others to do so, for ij $S$ Government purposes

The Los Alamos National Laboratory reauesis that the publisher identily this article as work pertormed under the auspices of the U S Department of Energy 


\title{
State Space Forecasting and Noise Reduction
}

\author{
S. Eubank, M. Casdagli, J. D. Farmer, J. Gibson \\ Los Alamos National Laboratory \\ Los Alamos, NM 87545
}

\begin{abstract}
We discuss the effects of nonlinearity and noise cn the problem of finding states and dynamics. We point out problems which arise in the construction of state space models for scalar time series. We describe solutions for the case when error due to noise dominates parameter estimation error and outline a framework for the general case.
\end{abstract}

\section{States and Dynamics}

A deterministic view of the world suggests that the best approach to modeling systems is to identify underlying states and their dyn mics. The two are closely related, since a state can be defined as that set of quantities in terms of which the dynamics is completely deterministic. The problem of finding states can be expressed as the problem of embedding time series data in a reconstructed state space. Thus recognizing the state variables for a system depends on finding good deterministic models for the dynamics in the embedding space.

Takens has proven that for generic dynamical systems, there is a diffeomorphism between the true state space and one reconstructed from delayed values of a scalar time series of observations on that system, as long as the dimension of the embedding space is large enough. Given noiseless data from a deterministic system and a method for modeling functions in high dimensional spaces, the determination of a good reconstruction for the state is not difficult: simply increase the dimension $m$ of the reconstruction and fit models for the dynamics. When a good model is obtained, the corresponding delay space is by definition a good embedding. A dimension algorithm such as Grassberger and Procaccia's gives a reasonable first estimate of $m$. [1] There are two problems with applying this strategy to real data: nonlinearity and noise.

\section{Nonlinearity And Noise}

If our models are drawn from a class of functions, e.g. linear functions, which does not include the true dynamics, forecasting arcuracy will be limited. We find that the best way to fit the graph of a nonlinear function in high dimensional space is with locally low order maps, much as splines rather than high order polynomials might be used to fit a one dimensional curve. The locality of the fits allows us to model a large class of dynamics without overparameterizing the models. $[2,3]$
In the presence of noise our definition of a state can be readily generalized by replacing "completely deterministic" with "as deterministic as possible". In this view, complete determinism is a limiting case of a Markov process in which the state transition probabilities are delta functions. The degree of determinism is measured by the width of the transition probability density. Since the error in a deterministic model is bounded below by this width, an optimal embedding is one which minimizes the width. The problems of noise reduction and optimal embedding are thus closely related.

In classical systems, we expect even noist: to be deterministically generated. The distinc:ion between noise and determinism is better thought of as a distinction between low and high dimet: sional dynamics. In this context, high means "higher than we are willing to model."

Even a small contamination by noise can invalidate Takens's theorem. For example, if the sampling rate $1 / \Delta$ is too large the change between consecutive measurements will be obscured by noise. In this case not all delay reconstructionis in $2 d+1$ dimensions will be sufficient for delor. mining the state, since the coordinates $x_{0}(t)$ and $x_{0}(t-\Delta)$ are redundant. Noise in a chaotic sistem makes the past seem causally disconnected from the future. That is, the measured values $x_{0}(t)$ and $x_{0}(t+k \Delta)$ are be statistically independent variables. In this case there is nothing to be gained by making the embedding dimention larger than $k-1$.

\section{Nonlinear Embeddings}

When the noise level is low and the amount of data large enough that distances between nearlyy points are smaller than the local radius of curvature of the dynamics, local principal components embeddings are optimal. These are local analogues of Broombead and King embed. dings, which project neighborhoods from in higl dimensional delay space onto lower-dimensionial hyperplanes.[4] The appropriate dimension for the hyperplanes is determined by a singular value analysis of the correlation matrix (on a local neighborhood. The projection averages orim noisy degrees of freedom to yield a beile! am. bedding than delay reconstruction in the same dimension. Figure 1 compares the acruracy of forecasts $\dot{\hat{x}}$ for noisy data from the Lorenz in. tractor using global and local principal compenent embeddings in three dimensions. Error is measured relative to the true (non-noisy) valm. 


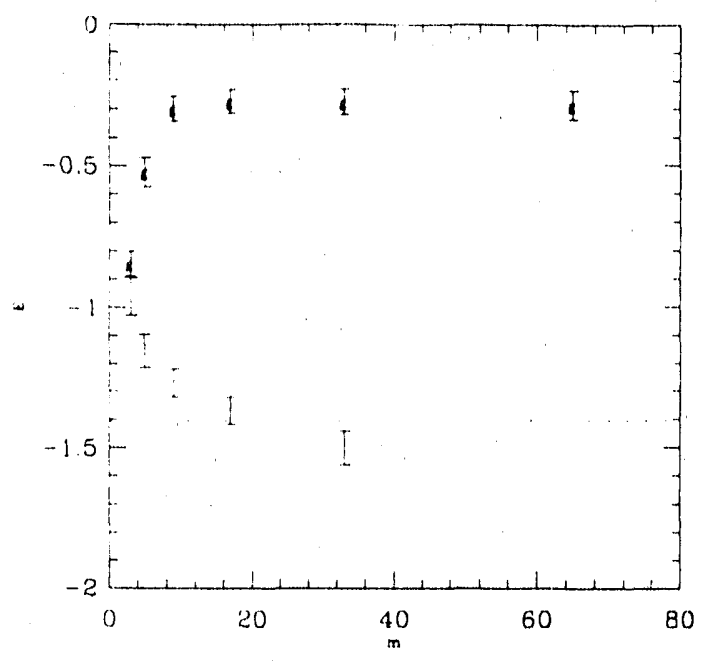

Figure 1: Forecast error using global (upper points) and local principal component embeddings $v s$. the number of dimensions in the original delay space. The dotted line shows the noise level in the original data.

of the time series $x(t)$ :

$$
E \equiv\left\langle\log \left(|x(t+T)-\hat{x}(t+T)| / \sigma_{x}\right)\right\rangle
$$

at $T=10$. The forecast error for 3 dimensional delay coordinates is -0.9 for this example. [5] For details of the technique and the conditions under which it performs well see the reference.

An alternative procedure is to use the estimated dynamics to push data measured at different times forward or backward to a common time. effectively vielding many measurements of a single variable. In addition, estimates of the relative precision of each measurement can be determined from the approximate dynamics. These cstimates give the weights with which the measurements should be averaged together.[2] Figure 2 shows the noise reduction achieved using this procedure when the true dynamics is known.

For higher noise levels, a nonlinear generalization of factor analysis would be useful. We outline below soine of the steps in such a method. Estimates of probability densities based on trial embeddings are used to build dynamical models which in turn help refine the embeddings.

Consider the problem of finding a transformation from $\left(x_{1}, x_{2}\right)$ to $\left(y_{1}, y_{2}\right)$, such that $y_{1}$ predicts a third variable $z$ as well as possible. A trpical linear solution uses Gram-Schmidt orthogonalization to enforce the following tiwo conditions: $y_{1}$ and $y_{2}$ are not redundant, and $y_{1}$ is maximally relevant to $z$. The second (variational) condition is equivalent to the (algebraic) condition that $y_{2}$ be irrelevant to $z$. In this framework, redundance and relevance are measurod by correlations.

These two conditions and the method of soIntion can be generalized to the nonlinear case.

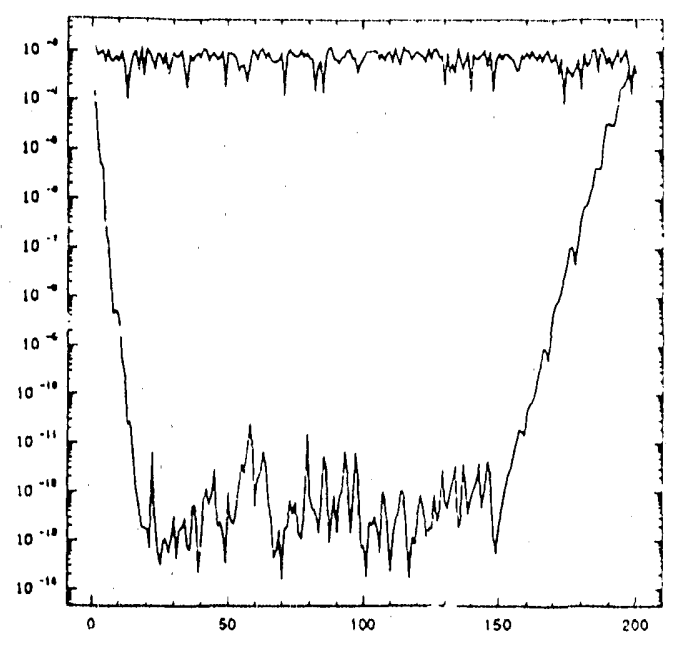

Fioure 2: Observational noise added to a time series is removed using knowledge of the dynamics. The noise in the original signal is shown in the upper curve; that of the cleaned signal in the lower curve.

Redundance is the amount of information about $z$ which is common to both $y_{1}$ and $y_{2}$. Vin. ables which are redundant can be combined io reduce noise. Redundance is best quantified not by a covariance as above, but by the independence of $y_{2}$ and $z$ given $y_{1}$. An information based statistic which measures redundance is $R \equiv I\left(z ; y_{1}\right) / I\left(z ; y_{1}, y_{2}\right)$, where the semicolon denotes mutual information. The second condition translates to maximizing the mutual information $I\left(z ; y_{1}\right)$ or minimizing $I\left(z ; y_{2}\right)$. A nonlinear generalization of Gram-Schmidt is likely to be useful for ellforcing these concitions: givon two variables $x_{1}$ and $x_{2}$ which are not statistically independent, they can be made so using the invertible transformation:

$$
\begin{aligned}
& y_{1}=x_{1}, \\
& y_{2}=\int_{-\infty}^{x_{2}} p_{x_{2} \mid x_{1}}(x) d x .
\end{aligned}
$$

\section{References}

[1] P. Grassberger and I. Procaccia. Physical Rrview Letters, 50:346, 1983.

[2] J.D. Farmer and J.J. Sidorowich. Exploiting chaos to predict the future and reduce noise. In Y.C. Lee editor, Evolution. Lerarning and Cognition, World Scientific, 1988.

[3] M. Casdagli. Nonlinear prediction of chantir time series. Physica D, 35:335, 1989.

[4] D.S. Broomhead and G.P. King. Extract. ing qualitative dynamics from experimential data. Physica $D, 20: 217,1987$.

[5] M. Casdagli, S. Eubank, J.D. Farmer, and J. Cibson. to appear in Physica D, 1990). 

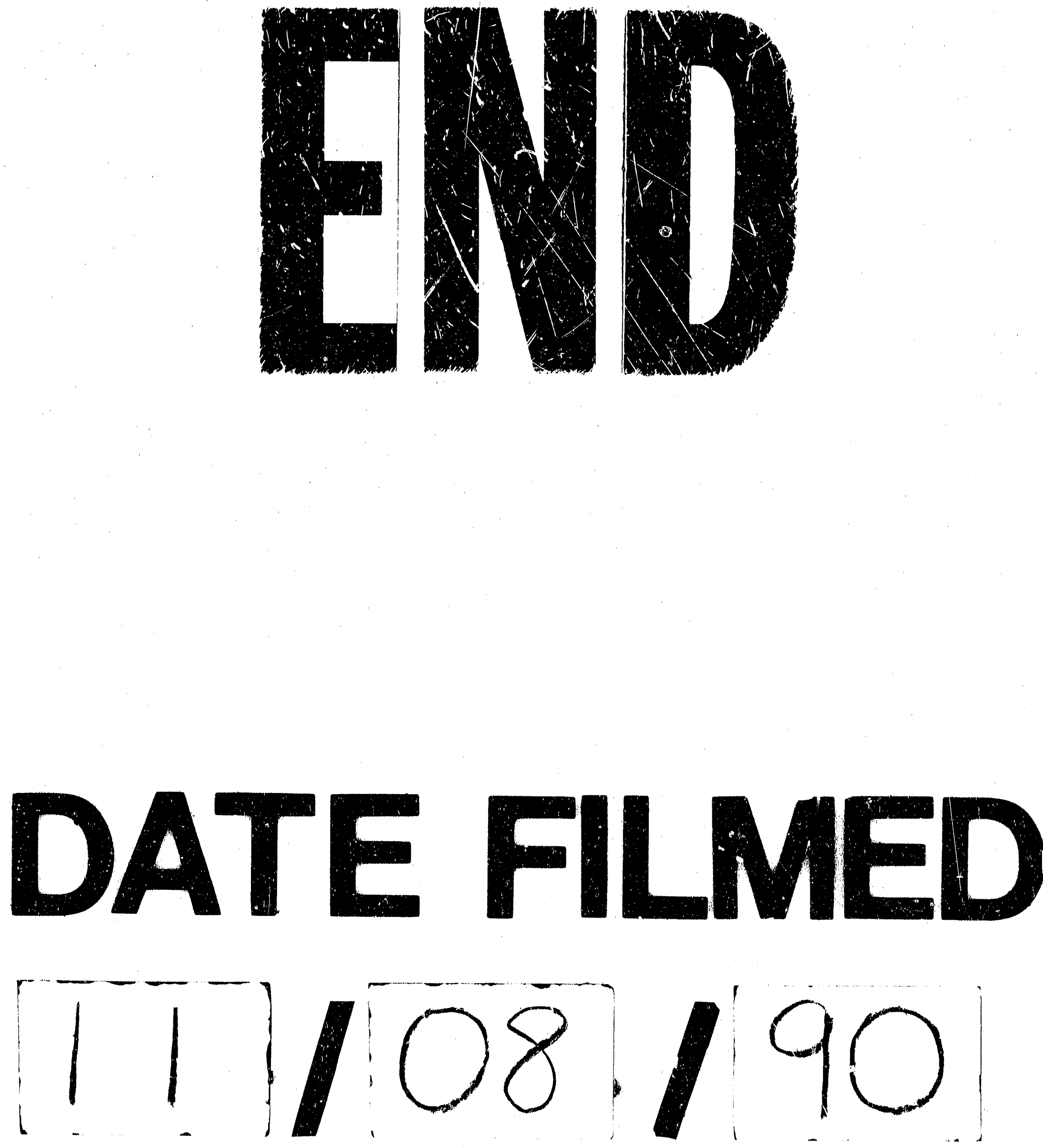
$\longrightarrow$ 